\title{
Development and evaluation of a bead-based Multiplexed Fluorescent ImmunoAssay (MFIA) for detection of antibodies to Salmonella enterica serogroup $B$ and $\mathrm{C} 1$ in pigs
}

\section{Sanne Schou Berger ( $\square$ sasbe@dtu.dk)}

Centre for Diagnostics, DTU Health Technology, Technical University of Denmark, DK-2800 Kgs. Lyngby Ulla Riber

Centre for Diagnostics, DTU Health Technology, Technical University of Denmark, DK-2800 Kgs. Lyngby Vibeke Frøkjaer Jensen

Centre for Diagnostics, DTU Health Technology, Technical University of Denmark, DK-2800 Kgs. Lyngby Klara Tølbøll Lauritsen

National Veterinary Institute, Technical University of Denmark, DK-2800 Kgs. Lyngby

\section{Lars Ole Andresen}

Centre for Diagnostics, DTU Health Technology, Technical University of Denmark, DK-2800 Kgs. Lyngby

\section{Research Article}

Keywords:

Posted Date: February 14th, 2022

DOI: https://doi.org/10.21203/rs.3.rs-1268456/v1

License: (c) (i) This work is licensed under a Creative Commons Attribution 4.0 International License. Read Full License 


\section{Abstract}

Background: Since 1995, a surveillance program for Salmonella has been applied in the Danish pig industry in order to reduce cases of human salmonellosis. The objective of this study was to develop a bead-based Multiplexed Fluorometric ImmunoAssay (MFIA) as an improved serological surveillance method compared to the Salmonella mix ELISA, which has been the national reference immunoassay in the Danish Salmonella surveillance program for about 20 years.

Results: An MFIA for detection of antibodies to Salmonella serogroup B and $\mathrm{C}_{1}$ was developed and optimized with regard to coupling of beads with Salmonella lipopolysaccharide antigens and establishing suitable assay conditions. The Salmonella MFIA was validated by testing sera from experimentally infected pigs as well as field sera from uninfected and infected pig herds, and by comparing to results from the Salmonella mix ELISA, which was run in parallel. Sensitivity and specificity was evaluated using receiver operating curve analysis showing an area under curve for the serogroup $B$ and $C_{1}$ MFIA of 0.984 and 0.998 , respectively. The Salmonella MFIA was shown to detect more antibody-positive samples in seropositive herds compared to the Salmonella mix ELISA, and Bayesian statistics confirmed that the MFIA had a considerably higher sensitivity (94.5\%) compared to the mix ELISA (75.1\%). The assay specificity was slightly lower for the Salmonella MFIA (96.8\%) compared to Salmonella mix ELISA (99.5\%). Coupled beads were stable for at least 1 year at $4^{\circ} \mathrm{C}$, and MFIA reproducibility and repeatability of the Salmonella MFIA were acceptable. Results from proficiency tests also indicated that the Salmonella MFIA was more sensitive than the Salmonella mix ELISA and that they had similar specificity.

Conclusions: A bead-based MFIA for simultaneous detection of porcine serum antibodies to Salmonella enterica serogroup $B$ and $C_{1}$ was developed and implemented in the Danish porcine serological Salmonella surveillance program in 2018. The Salmonella MFIA can distinguish, as opposed to the Salmonella mix ELISA, between antibodies to serogroup B and $\mathrm{C}_{1}$ and the MFIA shows considerably better sensitivity.

\section{Background}

Infections with bacteria belonging to the genus Salmonella enterica rarely cause clinical disease in pigs, but infected pigs form a major zoonotic reservoir. Especially Salmonella enterica serovar Typhimurium and serovar Infantis are known to be important and prevalent porcine pathogens that induce risk of severe infection and complications in human consumers. S. enterica subspecies are divided into serogroups based on similarities in the composition of their cell wall expressed LPS ( 0 antigens) and flagellar proteins (H antigens), as described in the White-Kauffman-Le Minor scheme [1].

In order to reduce human cases of salmonellosis, a surveillance program for Salmonella has been applied in the Danish pork industry since 1995 [2]. As part of the surveillance program, an Enzyme-Linked Immunosorbent Assay (ELISA) designated Salmonella mix ELISA was employed to screen porcine serum samples and meat juice for antibodies to Salmonella serogroup B and $\mathrm{C}_{1}$. The mix ELISA is based on the 
use of purified lipopolysaccharides (LPS) for detection of Salmonella serogroup specific antibodies. It was developed at the Danish National Veterinary laboratory and has since then been maintained in the laboratory as the national reference ELISA in the Danish porcine Salmonella surveillance program [3].

The LPS employed in Salmonella mix ELISA contains serogroup B specific 0:1,4,5,12 antigens from $S$. Typhimurium, and serogroup $C_{1}$ specific 0:6,7 antigens from $S$. Choleraesuis that are complementary to $S$. Infantis O-antigens [3]. Hence, Salmonella mix ELISA also detects antibodies against the remaining serovars included in the $B$ and $C_{1}$ serogroups. Due to the assay design of the Salmonella mix ELISA, it does not distinguish between antibodies to serogroup $B$ and $C_{1}$.

For decades, in-house ELISAs have also been used for diagnosis and surveillance of other diseases than salmonellosis in Danish pig herds, including those caused by Porcine Reproductive and Respiratory Syndrome Virus (PRRSV) and the bacterium Actinobacillus pleuropneumoniae [4-7]. Testing serum samples in separate ELISAs is resource demanding and time-consuming. In order to optimize this, a serological Multiplexed Fluorometric ImmunoAssay (MFIA) was developed and implemented based on the commercial XMAP technology platform developed by Luminex Corp., which can detect antibodies to multiple pathogens simultaneously. This assay setup utilizes magnetic polystyrene beads containing a combination of two fluorescent dyes that differentiate the beads into regions. Beads from these various regions are coated with antigens from different pathogens, making it possible to detect bound serum antibody with layers of biotinylated anti-porcine $\lg G$ and a fluorescent streptavidin-R-phycoerythrin (R-PE) reporter conjugate. The interaction between beads and sample antibodies is measured by flow cytometry, whereby the antigen is identified by the internal bead fluorescence and the level of bound sample antibodies is measured by intensity of the R-PE reporter.

In contrast to the Salmonella mix ELISA, a two-plex Salmonella MFIA offers the possibility of distinguishing between antibodies to Salmonella serogroup $B$ and $\mathrm{C}_{1}$. It also facilitates simultaneous detection of serum antibodies to other pathogens important in pig production, such as $A$. pleuropneumonia and PRRSV. A serological MFIA that detects and distinguishes between antibodies to seven serovars of $A$. pleuropneumonia in pigs has previously been developed, implemented, and described $[8,9]$. The additional serological MFIA that differentiate antibodies to PRRSV type 1 and PRRSV type 2 has recently been developed and implemented (manuscript in preparation).

Herein, we describe the development and validation of a bead-based multiplexed immunoassay that detects and distinguishes between antibodies against Salmonella serogroup B and $\mathrm{C}_{1}$ within a single serum sample volume.

\section{Results}

\section{Assay optimization and investigation of reagent stability}


Common assay conditions were identified for the Salmonella serogroup B and $\mathrm{C}_{1}$ MFIAs based on optimal signal-to-noise ratios. The same assay conditions were applicable to MFIAs for $A$.

pleuropneumoniae $[8,9]$ and PRRSV (manuscript in preparation), and all the assays could therefore be run in multiplex (data not shown). The serogroup $B$ and $\mathrm{C}_{1}$ assays were tested separately and in multiplex with the Quality Control (QC) panel, and antigen-specific reactivity with the QC panel was similar in the singleplex and multiplex format (data not shown).

From a shelf life study it was concluded that antigen-coupled beads maintained a stable interaction with serum antibodies for at least 13 months when stored at $4^{\circ} \mathrm{C}$ (Fig. 1). Fig. 1 shows the mean percent sample-to-positive ratios ( $\mathrm{S} / \mathrm{P} \%$ values) of the four serogroup $\mathrm{B}$ positive sera and the two serogroup $\mathrm{C}_{1}$ positive sera when tested over time. The results of the shelf life studies were used for estimating the between-run repeatability of the Salmonella MFIA. The percent coefficient of variance (CV\%) of the calculated S/P\% values were acceptable for each of the four serogroup B sera $(7.0 \%, 7.8 \%, 8.5 \%, 13.3 \%)$ and for the two serogroup $\mathrm{C}_{1}$ sera $(10.6 \%, 13.5 \%)$.

Within-run repeatability (within one plate) showed acceptable CV\% values (i.e. below $15 \%$ ) of $2.0 \%, 3.9 \%$, $4.9 \%$ and $13.3 \%$ for the four serogroup $B$ positive sera and $4.0 \%$ and $5.9 \%$ for the two serogroup $C_{1}$ positive sera.

The measurement of R-PE fluorescence intensity as a measure of sample antibody levels in the MFIA has a large linear range, although the validated Salmonella positive reference $(S / P \%=100)$, only uses around $35-40 \%$ of the estimated maximal signal. Therefore, highly positive samples may be calculated 3-4 times higher than the positive reference sample. To document linearity in the MFIA, serum samples with S/P\% > 300 were diluted in negative serum. Testing serial dilutions of such samples, showed linearity (Supplementary Fig. 1), which makes it possible to report samples with high levels of antibody directly, without pre-dilution. In contrast, ELISAs generally have a maximum optical density and a limited range of linearity, which may require pre-dilution of samples to determine "highly positive" samples correctly.

\section{MFIA validation}

ROC curve analysis was performed for Salmonella serogroup B MFIA using results from the validation with serum samples from naturally infected and uninfected pig herds. Results of the serogroup B MFIA were comparable to those of the mix ELISA with an area under the curve (AUC) of 0.984 (Fig. 2).

The cut-off for the serogroup B MFIA was six at the optimal differential positive rate (DPR), and the sensitivity and specificity at this cut-off was $96.6 \%$ and $94.5 \%$ respectively (Table 1 ).

The serogroup $C_{1}$ MFIA was also tested for reactivity with the 1425 samples in a duplex together with the serogroup $B$ MFIA, but only results with samples from experimentally infected pigs were used for ROC curve analysis, since the prevalence of serogroup $\mathrm{C}_{1}$ was very low in the tested herds. ROC curve analysis for the serogroup $\mathrm{C}_{1}$ showed comparable results to mix ELISA with an AUC of 0.998 (Fig. 2). At the 
optimal DPR, the cut-off for this assay was five with a sensitivity of $100 \%$ and a specificity of $98.5 \%$ (Table 1).

\section{Table 1}

Sensitivity, specificity and cut-off values for the Salmonella serogroup B and $\mathrm{C}_{1}$ MFIA

\begin{tabular}{|c|c|c|}
\hline & Serogroup B & Serogroup $C_{1}$ \\
\hline Optimal DPR* & 0,912 & 0.985 \\
\hline Cut-off & 6 & 5 \\
\hline Specificity $(\%)^{\star \star}$ & $96.6(95.0-97.7)$ & $100.0(94.5-100.0)$ \\
\hline 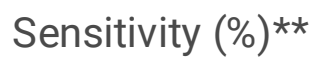 & $94.5(90.2-97.4)$ & $98.5(92.0-100.0)$ \\
\hline \multicolumn{3}{|c|}{ Adjusted cut-off values ${ }^{\star \star *}$} \\
\hline DPR & 0.896 & 0.940 \\
\hline Cut-off & 10 & 10 \\
\hline Specificity $(\%)^{\star \star}$ & $97,8(96.5-98.7)$ & $100.0(94.5-100.0)$ \\
\hline Sensitivity $(\%)^{\star \star}$ & $91.8(86.8-95.3)$ & $94.0(85.4-98.4)$ \\
\hline
\end{tabular}

For future use of the Salmonella MFIA in Danish swine herds, the cut-off values were adjusted to 10 in order to minimize the number of false positive reagents. Table 1 shows the specificities, sensitivities and DPR after cut-off adjustment.

Sensitivities, specificities and cut-off values are also indicated in a dot plot (Fig. 3), which shows reactivity in Salmonella MFIA with antibodies in serum samples that had negative or positive reaction in the Salmonella mix ELISA.

Fig. 4 shows pie charts that compare the results (using a cut-off of 10 for MFIA) of the 1425 samples tested in MFIA and mix ELISA at individual and herd level in relation to the herd status (uninfected or infected with Salmonella). In "uninfected" herds, MFIA detects $18.33 \%$ and mix ELISA detects $11.67 \%$ positive herds (Fig. 4C, 4D), while in "infected" herds, MFIA detects $64.44 \%$ positive herds and mix ELISA detects $46.67 \%$ positive herds (Fig. 4G, 4H). With MFIA, 30\% additional herds were therefore classified as positive compared to results obtained with mix ELISA.

If hypothesizing that samples which are negative in mix ELISA are true negatives, these would according to Table 2 represent false positive reactions when positive in MFIA. The relative risk of positive samples in MFIA, given a negative ELISA result was $11.38 \%$ for samples from the herds classified as infected and $0.68 \%$ for samples from the herds classified as non-infected (Table 2 ). 
Table 2

The risk of false positive results in MFIA in herds classified as Salmonella-infected and non-infected

\begin{tabular}{|c|c|c|c|c|c|}
\hline & & \multicolumn{2}{|c|}{$\begin{array}{l}\text { MFIA result for pigs that are negative in mix } \\
\text { ELISA }\end{array}$} & \multirow[t]{3}{*}{ Total } & \multirow{3}{*}{$\begin{array}{l}\text { Risk of MFIA } \\
\text { "false" positive } \\
\text { results (\%) }\end{array}$} \\
\hline & & $\begin{array}{l}\text { Salmonella positive } \\
\text { pigs in MFIA }\end{array}$ & $\begin{array}{l}\text { Salmonella negative } \\
\text { pigs }\end{array}$ & & \\
\hline & & & in MFIA & & \\
\hline \multirow{2}{*}{$\begin{array}{l}\text { Herd } \\
\text { classification }\end{array}$} & Infected & 74 & 576 & 650 & 11.38 \\
\hline & $\begin{array}{l}\text { Non- } \\
\text { infected }\end{array}$ & 4 & 587 & 591 & 0.68 \\
\hline
\end{tabular}

Data comprise only pigs classified as negative based on mix ELISA. The relative risk $(11.38 / 0.68)$ is 16.82.

If the hypothesis was true, we would have expected that the risk of an MFIA positive result for a mix ELISA negative sample would be similar whether or not the true negative sample was from herds classified as non-infected or infected. Here we have a highly significant difference of the risk (Fishers exact test: $p<0.001$, with a relative risk of 16.82) depending on herd class, and the hypothesis can therefore be rejected. The high relative risk indicates that a large proportion of the MFIA positive samples with a negative mix ELISA result, are most likely true positives, suggesting that MFIA data are more reliable for detecting positive herds. These results show that the specificity and sensitivity of the MFIA are higher than the estimates obtained with mix-ELISA as comparator.

Assay performance was further evaluated using Bayesian statistics, where the Hui-Walter test was performed on herds divided into two populations based on a "red/blue" classification system integrated into the Danish Specific Pathogen Free (SPF) system [10,11], where Salmonella prevalence is much higher in production herds ("blue" populations) than in breeding herds ("red" populations). The Hui-Walter test showed that MFIA had a considerably higher sensitivity of $94.5 \%$ compared to mix ELISA with a sensitivity of $75.1 \%$. Assay specificity was slightly lower for MFIA (96.8\%) compared to mix ELISA (99.5\%) (Table 3).

\section{Table 3}

Estimated sensitivities and specificities for MFIA and mix ELISA using Bayesian statistics (Hui-Walter test) 
Salmonella prevalence in production herd populations

Salmonella prevalence in breeding herd populations
MFIA

Mix ELISA
42.1

4.2

94.5

75.1

Specificity (\%)

MFIA

96.8

Mix ELISA

99.5

Proficiency test results provided by GD Deventer from year 2015 to 2020 (8 samples per proficiency test), showed that for some of the tested samples, MFIA had a higher sensitivity than mix ELISA. MFIA was more sensitive than mix ELISA for detection of antibodies to $S$. enterica serovar Brandenburg (serogroup B) and in contrast to MFIA, mix ELISA did not detect samples that were positive to $S$. enterica serovar Livingstone (serogroup $\mathrm{C}_{1}$ ). The antigens included in MFIA and mix ELISA are not specific for serogroup $C_{2}$ and serogroup $D_{1}$. Therefore, $S$. enterica serovar Goldcoast $\left(C_{2}\right)$ antibody positive samples were not detected positive in any of the proficiency tests, while it alternated whether the $S$. enterica serovar Panama $\left(D_{1}\right)$ antibody positive samples tested positive in both assays. The latter finding is caused by cross-reactivity, which is often observed between antibodies to serogroup $B$ and $D_{1}$ LPS antigens due to shared O-antigens (Table 4).

\section{Table 4}

Proficiency test results for samples tested from 2015 to 2020 in MFIA and Mix ELISA 


\begin{tabular}{|c|c|c|c|c|c|c|}
\hline \multirow[b]{2}{*}{$\begin{array}{l}\text { Salmonella } \\
\text { enterica serovar }\end{array}$} & \multirow[b]{2}{*}{ Serogroup } & \multirow[b]{2}{*}{$\begin{array}{l}0- \\
\text { antigen }\end{array}$} & \multirow[b]{2}{*}{$\begin{array}{l}\text { \# } \\
\text { samples } \\
\text { tested }\end{array}$} & \multicolumn{3}{|c|}{$\begin{array}{l}\text { Number of proficiency test samples correctly } \\
\text { identified as negative or positive }{ }^{\star}\end{array}$} \\
\hline & & & & $\begin{array}{l}\text { MFIA } \\
\text { serogroup B }\end{array}$ & $\begin{array}{l}\text { MFIA } \\
\text { serogroup } C_{1}\end{array}$ & $\begin{array}{l}\text { Mix ELISA } \\
\text { Serogroup } \\
B+C_{1}\end{array}$ \\
\hline Negative & - & - & 11 & 11 & 11 & 11 \\
\hline Brandenburg & B & $4,[5], 12$ & 8 & 8 & 0 & 4 \\
\hline Typhimurium & B & $\begin{array}{l}1,4, \\
{[5], 12}\end{array}$ & 14 & 14 & 0 & 14 \\
\hline Livingstone & $\mathrm{C}_{1}$ & $6,7,14$ & 6 & 0 & 6 & 0 \\
\hline Goldcoast** & $\mathrm{C}_{2}$ & 6,8 & 3 & 0 & 0 & 0 \\
\hline Panama ${ }^{\star \star \star}$ & $\mathrm{D}_{1}$ & $1,9,12$ & 6 & 3 & 0 & 3 \\
\hline \multicolumn{7}{|c|}{$\begin{array}{l}\text { * Sample ID is unknown to proficiency test participants, so samples may reoccur from } \\
\text { year to year. } \\
\text { ** The O-antigens used in MFIA and mix ELISA are not } 6,8 \text {. } \\
\text { *** Since the } \mathrm{D}_{1} \text { O-antigen is not included in the test, the reactants observed are due to } \\
\text { cross-reaction with the serogroup B O-antigen. }\end{array}$} \\
\hline
\end{tabular}

\section{Discussion}

When the Salmonella mix ELISA was included in the surveillance program in the mid-1990'ies, the intention of the Danish authorities was to use it as a serological screening tool that could supplement the less sensitive bacteriological cultivation methods [2]. It is important for a screening tool to have a high sensitivity and although the mix ELISA was a good solution at the time, more refined alternatives have since emerged, such as MFIAs represented by the Luminex XMAP Technology. MFIAs are known to include sensitive assays with high dynamic ranges [12-15].

In order to determine sensitivity and specificity of the developed Salmonella MFIA we used ROC curve statistical analysis where the MFIA was compared the Salmonella mix ELISA. However, comparison of a new assay with a reference assay, would result in false positives or negatives with the new test that are indeed true positives or negatives, if the new assay is more sensitive or specific than the reference assay. Consequently, the sensitivity and/or specificity of the new test will be underestimated. Therefore, in addition to using ROC curve analysis we also estimated sensitivity and specificity of the MFIA using the Hui-Walter paradigm, which is based on Bayesian statistics [16]. This paradigm is used to estimate 
sensitivity and specificity of two tests, when a definite "gold standard" reference assay is not available. Hereby it was shown that, despite using the same LPS antigen preparations for detection of antibodies, the developed MFIA is more sensitive than mix ELISA, detecting more Salmonella antibody-positive samples in seropositive herds.

By facilitating testing for and differentiation of multiple targets, Luminex technology is highly suitable for screening populations for antibodies to multiple targets using a single sample volume. In the Salmonella mix ELISA, two LPS antigens (serogroup $B$ and $C_{1}$ specific) are coupled to the bottom of the same wells, which may introduce an interference so that antigens are not presented optimally or equally. This interference is not an issue in bead-based MFIA, since different subsets of beads are coupled with different antigens. Thus, a higher sensitivity of the Salmonella MFIA compared to Salmonella mix ELISA probably also originate from a more optimal antigen presentation on the surface of beads. Importantly, the presentation of Salmonella antigens on separate bead subsets in the MFIA provides a major diagnostic advantage compared to the Salmonella mix ELISA, since the MFIA allows distinction between antibodies to serogroup $B$ and $\mathrm{C}_{1}$.

The cut-off values for the Salmonella serogrup B and $C_{1}$ MFIAs were adjusted to 10 to increase specificity, in order to reduce the probability of getting false positive results. False positive results in the Danish surveillance system can have severe and unnecessary consequences for the individual swine farm, including trading restrictions and demands for resampling and retesting. Since the tested sample size from each herd is normally 10 (or even higher when sampling for antibody profiles in a pig herd), and given that the sensitivity is acceptable even after increasing the specificity, the surveillance is still highly sensitive with the new cut-off, with regards to detecting Salmonella antibodies in truly positive herds.

The Salmonella MFIA has been successfully employed in the Danish surveillance program since 2018. Identical assay conditions are used in all our in-house developed MFIAs for porcine serum samples, which permit simultaneous analysis for Salmonella serogroup $\mathrm{B}$ and $\mathrm{C}_{1}$, Actinobacillus pleuropneumoniae serovar 1, 2, 5, 6, 7, 10 and $12[8,9]$ and PRRSV type 1 and 2 (manuscript in preparation). In addition, a subset of the MFIAs can be run according to customer requests. The detection of antibodies to multiple analytes within a serum sample reduces the required amount of serum sample, as well as the amount of time and labor needed to test the samples. This shortens the response time and significantly lowers the costs for serological testing and surveillance.

Future MFIAs for other targets could include Salmonella assays for detection of antibodies in porcine sera to serogroups other than $B$ and $C_{1}$ that include serovars with zoonotic potential. Furthermore, there may be a potential to include serological testing for other important pathogens in the pig production.

\section{Conclusion}

In conclusion, the serological bead-based MFIA described here for detection of antibodies to Salmonella serovars belonging to serogroup $\mathrm{B}$ or $\mathrm{C}_{1}$ has a good repeatability and a high sensitivity and specificity 
compared to the alternative; the Salmonella mix ELISA. To our knowledge, this is the first report of an MFIA for simultaneous detection of antibodies to different serogroups of Salmonella enterica in pigs.

\section{Methods}

\section{Serum samples}

For assay optimizations, testing assay repeatability as well as the shelf life of coupled beads, a serum panel was used. This panel contained four sera positive for antibodies to Salmonella serogroup B (separate sera with different levels of antibodies to $S$. Typhimurium), two sera positive for antibodies to Salmonella serogroup $C_{1}$ (one with antibodies to $S$. Cholerasuis and one with antibodies to $S$. Infantis), as well as a Salmonella negative serum pool. These sera originated from experimental Salmonella infection studies performed decades ago at the National Veterinary Laboratory, Technical University of Denmark, and for years used as part of a QC serum panel for routine evaluation of the performance of ELISAs measuring Salmonella antibodies to serogroup B and $\mathrm{C}_{1}[3]$.

For assay validation, two samples from the serum panel that were positive for antibodies to either serogroup $B$ and $C_{1}$ were pooled, and this serum pool was used as a combined positive control sample applied to each assay plate during the validation procedure along with serum from a Salmonella antibody-negative pig.

Field samples used for the final validation of the Salmonella MFIA included 1425 samples collected in 2016 from 105 Danish pig herds participating in the Salmonella surveillance program with routine ELISA screening, as well as samples tested for serological diagnosis of Salmonella infection in pig herds. In 2016, immediately prior to sampling, the herds had been categorized in the Danish herd health status database as Salmonella free (status: uninfected) or as previously infected with Salmonella (status: infected).

Since Salmonella serovars belonging to serogroup $\mathrm{C}_{1}$ are rare in Denmark, serum samples, obtained from two previous studies with experimentally infected pigs, performed at the National Veterinary laboratory, were used for the validation. One study was from 1998/1999 with 12 pigs inoculated with S. enterica serovar Infantis, and another study from 2000/2001 with six pigs inoculated with S. enterica serovar Choleraesuis (both unpublished).

Proficiency tests for serological porcine Salmonella testing were provided by GD Deventer, the Netherlands from the year 2015 to 2020 and included 8 freeze-dried serum samples per year.

\section{Preparation of lipopolysaccharide}

Lipopolysaccharide (LPS) antigen from Salmonella enterica serovar Typhimurium strain no. 3389-1/92 and Salmonella enterica serovar Choleraesuis var. Kunzendorf strain no. 143 were prepared after 
cultivation in a bioreactor and purified by extraction with hot phenol as previously described [17]. These LPS antigen preparations are also used as detection antigens in the Salmonella mix ELISA.

\section{Coupling of lipopolysaccharide to magnetic beads}

LPS preparations from Salmonella serovars belonging to serogroup B (LPS from $S$. Typhimurium) and $\mathrm{C}_{1}$ (LPS from S. Cholerasuis) were coupled to separate subsets of magnetic beads (Bio-Plex Pro magnetic $\mathrm{COOH}$ beads, Luminex) using a method described previously $[8,18]$. Briefly, $1.25 \times 10^{7}$ beads were resuspended in $250 \mu \mathrm{L}$ of $0.1 \mathrm{M}$ 2-( $\mathrm{N}$-morpholino)ethanesulfonic acid (MES buffer; $\mathrm{pH}$ 5.0; M 22933, Sigma-Aldrich, St. Louis, MO) and then vortexed (10 s) and sonicated (20 s; Sonorex Digitec, Bandelin, Berlin, Germany). A volume of $750 \mu \mathrm{L}$ LPS diluted in MES buffer and $25 \mu \mathrm{L}$ of fresh $N$-(3dimethylaminopropyl)- $N$-ethylcarbodiimide hydrochloride (EDC; 03449, Sigma-Aldrich) solution (50 $\mathrm{mg} / \mathrm{mL}$ ) was added. After vortexing, the solution was incubated for $40 \mathrm{~min}$ in the dark at room temperature (RT) in a rotator (PTR-35, Grant Instruments, Cambridge, England). An additional $1 \mathrm{~mL}$ of LPS, together with $25 \mu \mathrm{L}$ fresh EDC, was added and incubated with the beads for 40 min in the dark at RT in the rotator. The last incubation step was repeated for a total of 3 incubations with LPS. A magnet (DynaMag-5, Thermo Fisher Scientific, Waltham, MA) for 5-mL tubes (Eppendorf Protein LoBind tube, Sigma-Aldrich) was used for washing the beads (3 times) with $3 \mathrm{~mL}$ of phosphate-buffered saline (PBS), $0.1 \%$ bovine serum albumin (BSA), $0.02 \%$ Tween 20 , and $0.05 \%$ sodium azide (PBS-TBN; $\mathrm{pH} 7.4$ ). The beads were stored in the dark at $2-8^{\circ} \mathrm{C}$ in $1 \mathrm{~mL}$ of PBS-TBN. The coupling procedures were optimized with regard to amount of antigen, buffers, incubation time as well as conditions providing the highest signal-to-noise ratio of the median fluorescent intensities (MFIs) measured after testing the beads with serum samples in the process of assay development.

\section{Multiplexed Fluorometric ImmunoAssay (MFIA)}

Suspensions of beads coupled with LPS from serogroup $B$ and $C_{1}$, respectively, were vortexed $(10 \mathrm{sec})$ and mixed at a concentration of $8 \times 10^{4}$ beads $/ \mathrm{mL}$ per bead subset in a single volume of assay buffer $A$ (PBS $0.05 \mathrm{M}, 0.05 \%$ Tween, 1\% BSA, $0.5 \mathrm{M} \mathrm{NaCl}$ ). After vortexing (10 sec) and sonication (20 sec), $25 \mu \mathrm{L}$ of bead suspension was added to black flat-bottom, 96-well plates (Bio-Plex Pro, Bio-Rad, Hercules, CA) together with $25 \mu \mathrm{L}$ of pig serum diluted in assay buffer $A$ (final serum dilution, 1:200). All incubations were performed at RT in the dark (covered with aluminum foil) on a rotating shaker (MTS 2/4 digital microtiter shaker, IKA-Werke, Staufen, Germany). The plates were incubated for $60 \mathrm{~min}$ and washed with wash buffer (PBS, 0.05\% Tween) using an automated plate washer for magnetic beads (ELx405, BioTek, Winooski, VT). Next, $25 \mu \mathrm{L}$ biotin-conjugated rabbit anti-swine IgG (SAB3700429, Sigma-Aldrich) was added per well ( $2 \mu \mathrm{g} / \mathrm{mL}$, diluted in assay buffer B (PBS, $0.05 \%$ Tween, $1 \% \mathrm{BSA})$ ), and incubated with the beads for $30 \mathrm{~min}$. The plates were washed in the automated plate washer, and incubated for 30 min with $75 \mu \mathrm{L}(1 \mu \mathrm{g} / \mathrm{ml}$, diluted in assay buffer B) of Streptavidin-R-Phycoerythrin (S21388, Thermo Fisher Scientific). The plates were shaken on the rotating shaker for $30 \mathrm{sec}$, and samples were read in a Bio-Plex 
200 flow cytometric platform (Bio-Rad) adjusted to acquire a $50-\mu \mathrm{L}$ sample and count a minimum of 50 beads/analyte. During validation, all samples were analyzed in duplicates. Bound pig serum IgG was measured as the MFI signal of R-phycoerythrin (R-PE) after exclusion of aggregated beads by gating. Data were acquired using the flow cytometric platform software (Bio-Plex Manager software v.6.1, BioRad).

\section{Assay optimization and test of reagent stability}

Parameters including assay reaction time, washing steps, buffer composition, incubation temperature as well as antigen-, serum-, and conjugate concentrations were optimized in single-plex assays using serum samples in the QC panel.

To evaluate the shelf life of coupled beads over a 13-month period duplicates of the samples included in the QC serum panel were tested monthly by two different operators (Fig. 1). Results of the shelf life validation studies were also used for measuring between-run repeatability of the analysis. A within-run repeatability study was performed with 12 separate dilutions of the samples from the QC serum panel that were run on the same assay plate. Assay repeatability was defined as the percent mean coefficient of variation of the MFI values (CV\%).

\section{Data analysis}

Percent sample-to-positive ratios (S/P\%) were calculated using the following formula:

$$
\mathrm{S} / \mathrm{P} \%=\frac{\left(\mathrm{MFI} \text { sample }-\mathrm{MFI}^{-} \text {negative control }\right)}{\left(\mathrm{MFI} \text { positive control }-\mathrm{MFI}^{\mathrm{M}} \text { negative control }\right)} \times 100 \%
$$

Receiver operating characteristic (ROC) curve analysis was used for the determination of test analysis quality compared to the existing in-house mix ELISA. Differential positive rates (DPR) (defined as sensitivity + specificity -1 ) were used together with dot plot correlation curves and $2 * 2$ contingency tables to determine cut-off values for the individual analyte in the MFIA [19-21].

The statistical programs R and OpenBUGS were used for the Hui-Walter test.

\section{Assay validation}

After optimization of the Salmonella multiplex assay, beads coupled with serogroup B and $C_{1}$ LPS antigens were mixed and tested in a two-plex assay against 1425 field serum samples from 105 seropositive or seronegative SPF herds. The same samples were tested concurrently in Salmonella mix 
ELISA [3] which is the national reference assay for surveillance of Salmonella in pigs and for herd classification in the Danish SPF system $[10,11]$.

\section{Receiver Operating Curve analysis}

In ROC curve statistical analysis, if a herd was found positive in the Salmonella mix ELISA, MFIA data were included for the positive sera while samples in the same herd that tested negative in the Salmonella mix ELISA were excluded in order to minimize the influence of animals undergoing seroconversion, which would give borderline reactions. Therefore, of the 1425 tested serum samples only 953 were included in the ROC curve analysis.

Since Salmonella serovars belonging to serogroup $\mathrm{C}_{1}$ are rare in Denmark, ROC-curve analysis for this serogroup was performed on results obtained by testing samples from naïve and experimentally infected pigs originating from two studies with samples before inoculation, and samples taken with regular intervals after inoculation. Samples taken during seroconversion (day 4-7 post inoculation) were not included in ROC curve analysis since these can show borderline reactions. Samples taken before seroconversion (before inoculation and day 1-3 post inoculation) were used as negative samples $(n=65)$, while samples taken after seroconversion were used as positive samples $(n=67)$.

\section{Bayesian statistics}

The Hui-Walter paradigm, which is based on Bayesian statistics can be used to estimate sensitivity and specificity in the absence of a definite "gold standard" [16]. The test is based on data from both assays applied to two (sub) populations with different prevalences. Here, we used two populations defined in the Danish SPF system based on different biosecurity measures and surveillance of specific porcine infectious diseases $[10,11]$. Whereas the "blue" herds are mainly production herds with a high level of biosecurity, the "red" herds are breeding herds with the highest level of biosecurity. Division into herds with a status of 'infected' or 'non-infected' in the Salmonella surveillance system was not applied for the Hui-Walter test, because it would have introduced a statistical bias since it is based on results from the Salmonella mix ELISA [3].

\section{Abbreviations}

AUC: Area Under Curve

BSA: Bovine Serum Albumin

CV\%: Percent Coefficient of Variation

DPR: Differential Positive Ratio 
ELISA: Enzyme-Linked Immunosorbent Assay

LPS: Lipopolysaccharide

MES: 2-(N-morpholino)ethanesulfonic acid

MFI: Median Fluorescence Intensity

MFIA: Multiplexed Fluorescent ImmunoAssay

PRRSV: Porcine Reproductive and Respiratory Syndrome Virus

QC: Quality Control

ROC: Receiver Operating Curve

R-PE: R-PhycoErythrin

RT: Room Temperature

S/P\%: Percent Sample-to-Positive ratio

SPF system: Specific Pathogen Free system

\section{Declarations}

\section{Ethics}

Serum samples used for the validation of the MFIA had previously been collected for the Danish Salmonella surveillance program. None of these samples were extraordinarily taken from animals.

The serum samples from experimentally infected animals were historical samples, originating from previous infection studies (unpublished) performed at the Veterinary Institute, Technical University of Denmark. These animal experiments had been performed in accordance with guidelines and regulations and had been approved by the Danish Animal Experiments Inspectorate.

\section{Consent for publication}

All authors of the manuscript have read and agreed to its content and are accountable for all aspects of the accuracy and integrity of the manuscript.

\section{Availability of data and materials}


The datasets generated and/or analyzed during the current study are not publicly available due to the fact that swine herds may be identified from the datasets, but are available from the corresponding author in a redacted form on reasonable request.

\section{Competing interests}

The authors declare no competing interests or other interests that might be perceived to influence the results and/or discussion reported in this paper.

\section{Funding}

The authors received no financial support for the research, authorship, and/or publication of this article.

\section{Authors' contributions}

SSB, UR, KTL and LOA conceived and designed the study. SSB performed laboratory experiments. SSB, VFJ, UR and LOA analyzed the results. All authors wrote and approved the final manuscript.

\section{Acknowledgments}

We thank the laboratory staff at Centre for Diagnostics, Health Technology, Technical University of Denmark. Especially, thanks to Natascha Morton, Liselotte Koch Nielsen, Henriette Vorsholt, and Jonathan Rogersen for excellent technical laboratory assistance.

\section{References}

1. Grimont PAD, Weill F-X. Antigenic formulae of the Salmonella serovars. WHO Collaborating Centre for Reference and Research on Salmonella at Institute Pasteur in France, Paris. $9^{\text {th }}$ Edition. 2007:150 p.

2. Alban L, Baptista FM, Møgelmose V, Sørensen LL, Christensen H, Aabo S, et al. Salmonella surveillance and control for finisher pigs and pork in Denmark - A case study. Food Res. Int. 2012;45(2):656-665.

3. Nielsen B, Baggesen D, Bager F, Haugegaard J, Lind P. The serological response to Salmonella serovars typhimurium and infantis in experimentally infected pigs. The time course followed with an indirect anti-LPS ELISA and bacteriological examinations.Vet. Microbiol. 1995;47(3-4):205-218.

4. Klausen J, Ekeroth L, Grøndahl-Hansen J, Andresen LO.An indirect enzyme-linked immunosorbent assay for detection of antibodies to Actinobacillus pleuropneumoniae serovar 7 in pig serum. J. Vet. Diagn. Invest. 2007;19:244-249. 
5. Klausen J, Andresen LO, Barfod K, Sørensen V. Evaluation of an enzyme-linked immunosorbent assay for serological surveillance of infection with Actinobacillus pleuropneumoniae serotype 5 in pig herds. Vet. Microbiol. 2002;88(3):223-232.

6. Grøndahl-Hansen J, Barfod K, Klausen J, Andresen LO, Heegaard PMH, Sørensen V. Development and evaluation of a mixed long-chain lipopolysaccharide based ELISA for serological surveillance of infection with Actinobacillus pleuropneumoniae serotypes 2, 6 and 12 in pig herds. Vet. Microbiol. 2003;96(1):41-51.

7. Sørensen KJ, Strandbygaard B, Bøtner A, Madsen ES, Nielsen J, Have P. Blocking ELISA's for the distinction between antibodies against European and American strains of porcine reproductive and respiratory syndrome virus. Vet. Microbiol. 1998;60(2-4):169-177.

8. Berger SS, Lauritsen KT, Boas U, Lind P, Andresen LO. Simultaneous detection of antibodies to five Actinobacillus pleuropneumoniae serovars using bead-based multiplex analysis. J. Vet. Diagn. Invest. 2017;29(6):797-804.

9. Boas U, Sørensen MB, Andresen LO, Berger SS. A generic protocol to immobilize lipopolysaccharides on microbeads for multiplex analysis. Anal. Methods. 2021;13(22):2485-2494.

10. Specific Pathogen Free System (SPF - Danmark). https://www.spf.dk. Accessed 6 Jan 2022.

11. Antunes ACL, Halasa T, Lauritsen KT, Kristensen CS, Larsen LE, Toft N. Spatial analysis and temporal trends of porcine reproductive and respiratory syndrome in Denmark from 2007 to 2010 based on laboratory submission data. BMC Vet. Res. 2015;11:303.

12. Wang $\mathrm{H}$, Cong F, Guan J, Xiao L, Zhu Y, Lian Y, et al. Establishment of XMAP for the simultaneous detection of antibodies to Newcastle disease virus and avian influenza virus. Poult. Sci. 2019;98(3):1494-1499.

13. Baker HN, Murphy R, Lopez E, Garcia C. Conversion of a capture ELISA to a Luminex xMAP assay using a multiplex antibody screening method. J. Vis. Exp. 2012;(65):e4084, doi:10.3791/4084.

14. Yu C, Yang C, Song S, Yu Z, Zhou X, Wu J. Development of a sensitive Luminex xMAP-based microsphere immunoassay for specific detection of Iris yellow spot virus. Virol. J. 2018;15:62.

15. Ayouba A, Touré A, Butel C, Keita AK, Binetruy F, Sow MS, et al. Development of a sensitive and specific serological assay based on Luminex technology for detection of antibodies to Zaire Ebola virus. J. Clin. Microbiol. 2017;55(1):165-176.

16. Johnson WO, Gastwirth JL, Pearson LM. Screening without a "gold standard": The Hui-Walter paradigm revisited. Am. J. Epidemiol. 2001;153(9):921-924. https://doi.org/10.1093/aje/153.9.921.

17. Wiuff C, Jauho ES, Stryhn H, Andresen LO, Thaulov K, Boas U, et al. Evaluation of a novel enzymelinked immunosorbent assay for detection of antibodies against Salmonella, employing a stable coating of lipopolysaccharide-derived antigens covalently attached to polystyrene microwells. J. Vet. Diagn. Invest. 2000;12(2):130-135.

18. lihara H, Niwa T, Monir Shah M, Hong Nhung P, Xiao Song S, Hayashi M, et al. Rapid multiplex immunofluorescent assay to detect antibodies against Burkholderia pseudomallei and taxonomically closely related nonfermenters. Jpn. J. Infect. Dis. 2007;60(4):230-234. 
19. Gardner IA, Greiner M. Receiver-operating characteristic curves and likelihood ratios: improvements over traditional methods for the evaluation and application of veterinary clinical pathology tests. 2006;35(1):8-17.

20. Zweig MH, Campbell G. Receiver-operating characteristic (ROC) plots: a fundamental evaluation tool in clinical medicine. Clin. Chem. 1993;39(4):561-577.

21. World Organization for Animal Health (OIE). Principle and methods of validation of diagnostic assays for infectious diseases. Manual of diagnostic tests and vaccines for terrestrial animals. 2018:72-

87.Https://www.oie.int/fileadmin/Home/eng/Health_standards/tahm/1.01.06_VALIDATION.pdf Assessed 6 Jan 2022.

\section{Figures}

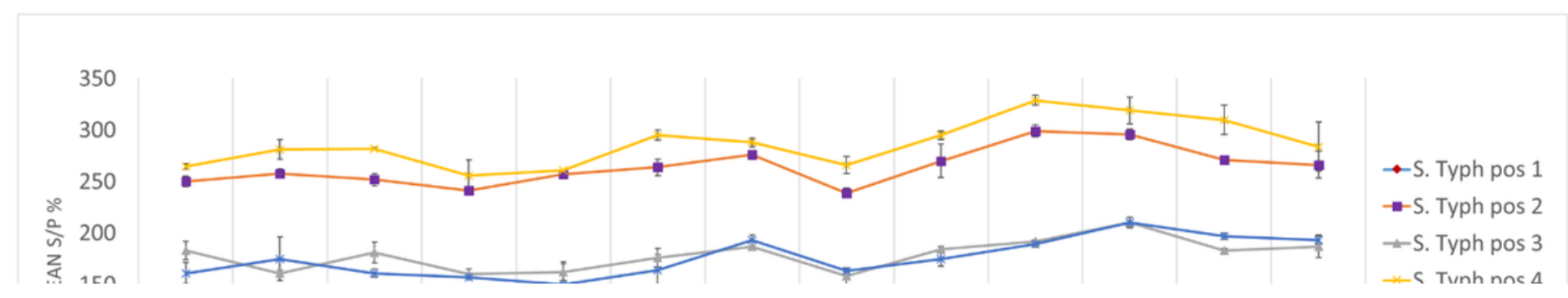

\section{Figure 1}

Time-dependent activity of beads coupled with LPS from Salmonella serogroup B and $\mathrm{C}_{1}$. Antigencoupled beads were tested with the Quality Control (QC) panel after 1 day and then monthly for one year. Bead activity was calculated as an S/P\% value. The plot shows mean S/P\% values \pm STD for each of the four serogroup B positive sera ( $S$. Typhimurium) and the two serogroup $C_{1}$ sera $(S$. Infantis and $S$. Choleraesuis). 

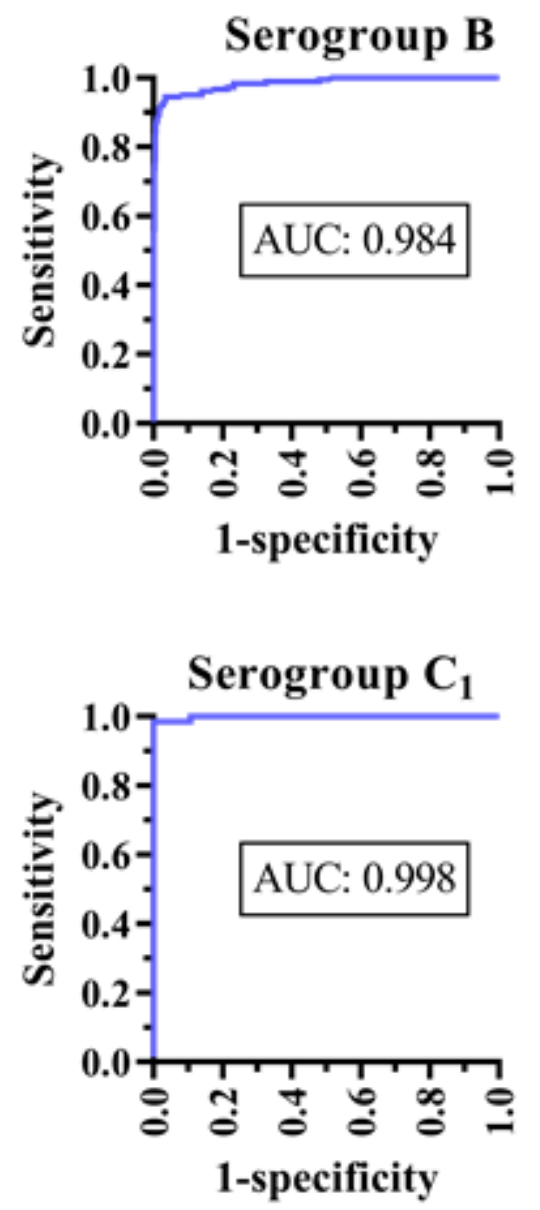

Figure 2

Receiver Operating Curves (ROCs) for Salmonella serogroup B and $\mathrm{C}_{1}$ multiplex-analysis with Area Under Curve (AUC) shown in each graph. The reference assay was an in-house Salmonella mix ELISA. 

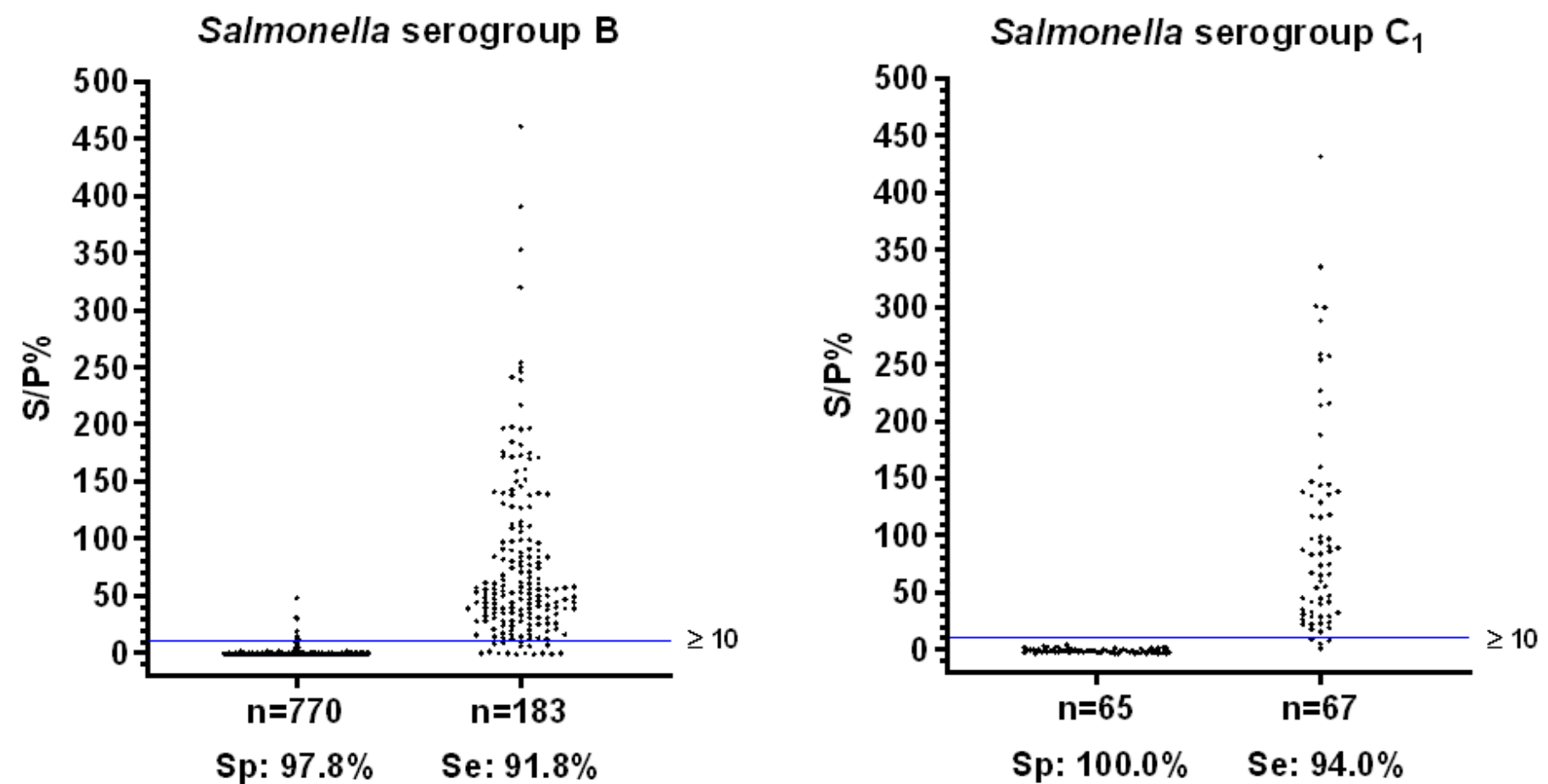

Figure 3

Dot plots with results from the validation of the Salmonella serogroup B and $\mathrm{C}_{1}$ MFIA. Reactivity with antibodies in serum samples from Salmonella mix ELISA-negative pigs (left) and Salmonella mix ELISApositive pigs (right). Horizontal lines indicate the adjusted cut-off values for each analysis. Below each graph are shown specificities (Sp) and sensitivities (Se) at the specified cut-off. S/P\% = percent sampleto-positive ratio. 


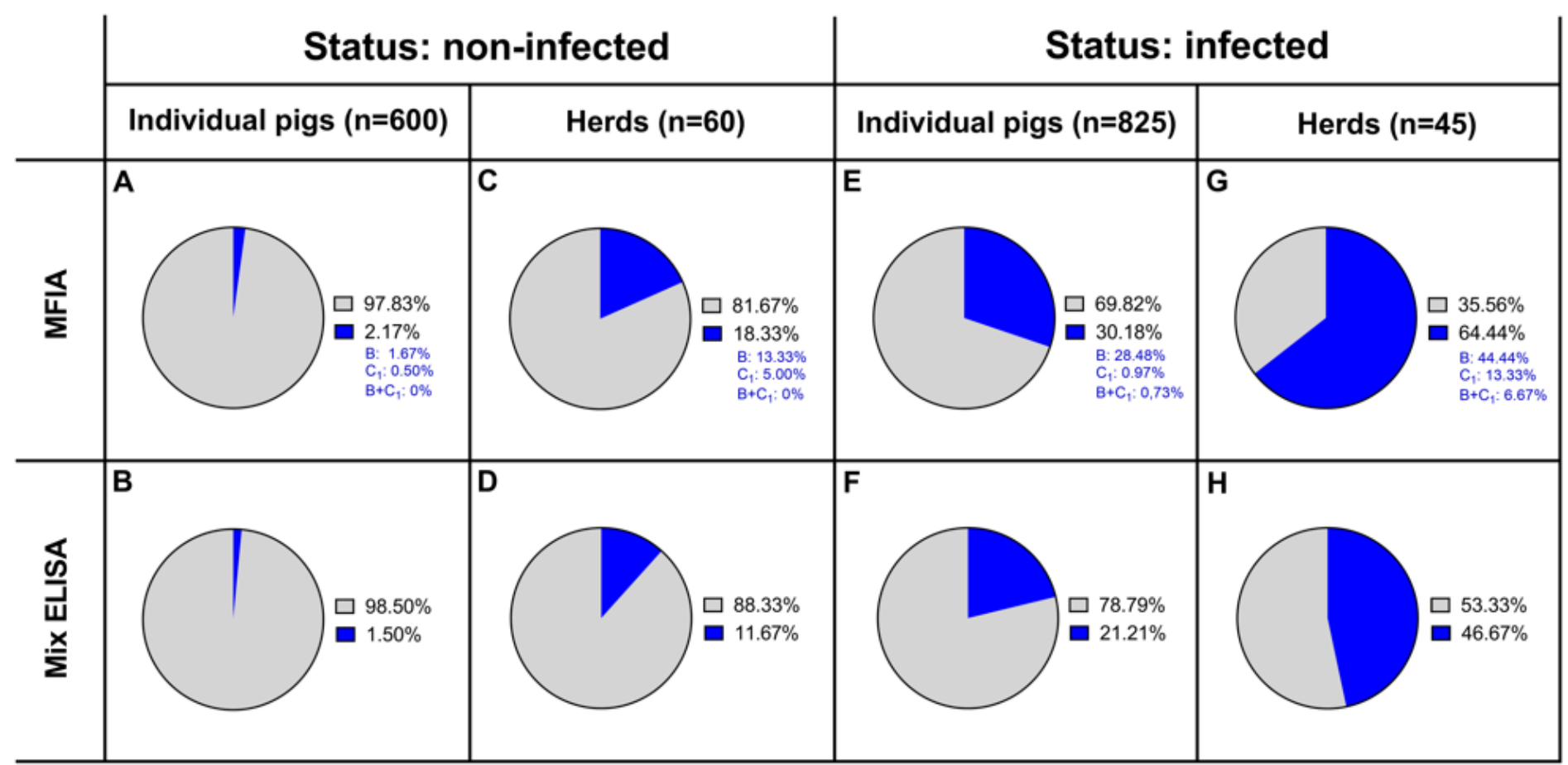

Figure 4

Percentages of 1425 swine samples from 105 herds that tested negative (grey) or positive (blue) in MFIA and mix ELISA for antibodies to Salmonella enterica serogroup $B$ and $\mathrm{C}_{1}$ in herds previously categorized with a status as Salmonella free (A-D) or Salmonella infected (E-H). Since MFIA distinguishes between serogroup $B$ and $C_{1}$, percentage of pigs or herds infected with the different serogroups are indicated with blue text.

\section{Supplementary Files}

This is a list of supplementary files associated with this preprint. Click to download.

- Suppl.fig.1.docx 